\title{
Thermodynamic signatures for topological phase transitions to Majorana and Weyl superfluids in ultracold Fermi gases
}

\author{
Kangjun Seo, ${ }^{1}$ Chuanwei Zhang, ${ }^{2}$ and Sumanta Tewari ${ }^{1}$ \\ ${ }^{1}$ Department of Physics and Astronomy, Clemson University, Clemson, SC 29634, USA \\ ${ }^{2}$ Department of Physics, the University of Texas at Dallas, Richardson, TX 75080 USA
}

\begin{abstract}
We discuss the thermodynamic signatures for the topological phase transitions into Majorana and Weyl superfluid phases in ultracold Fermi gases in two and three dimensions in the presence of Rashba spin-orbit coupling and a Zeeman field. We analyze the thermodynamic properties exhibiting the distinct nature of the topological phase transitions linked with the Majorana fermions (2D Fermi gas) and Weyl fermions (3D Fermi gas) which can be observed experimentally, including pressure, chemical potential, isothermal compressibility, entropy, and specific heat, as a function of the interaction and the Zeeman field at both zero and finite temperatures. We conclude that among the various thermodynamic quantities, the isothermal compressibility and the chemical potential as a function of the artificial Zeeman field have the strongest signatures of the topological transitions in both two and three dimensions.

PACS numbers: 03.75.Ss, 67.85.Lm, 67.85.-d
\end{abstract}

\section{INTRODUCTION}

Ultracold fermionic quantum gases have many advantages for investigations of quantum phenomena in a highly tunable way. The experimental ability to control the interaction between the Fermi atoms via Feshbach resonances [1] enables to study the crossover phenomena [2] from the BCS superfluid of weakly bound Cooper pairs to the BEC of tightly bound molecules [3], universal properties in the unitary regime [4], vortices [5], and so forth. In addition, the experimental control of the internal spin states via radio-frequencies provides the study of the emergence of new superfluid phases in a Fermi gas with population imbalance which serves as an effective Zeeman field [6-8]. Another new development enables to trap two dimensional fermions in the quantum degenerate regime [9, 10].

Furthermore, in recent experimental breakthroughs the spin-orbit coupling (SOC) has been developed using twophoton Raman processes in bosonic as well as fermionic gases [11-15], which has given rise to theoretical investigations on the new quantum states of matter [16- 19$]$. Thus far, the experimental realization of SOC is an equal combination of Rashba [20] and Dresselhaus [21] types of SOC. It has been shown theoretically that in the presence of Rashba SOC and a perpendicular Zeeman field, 2D cold fermion systems with an $s$-wave Feshbach resonance can enter into a topological superfluid phase with zero energy Majorana fermion excitations localized at the order parameter defects such as vortices [18, 22, 23]. It has also been shown that in 3D the corresponding system is capable of supporting the intriguing Weyl fermion excitations [17]. The realization of ultra-cold fermionic gases with Rashba SOC and a Zeeman field thus offers the tantalizing possibility of experimental engineering of all three kinds of fundamental relativistic fermions, namely, the Dirac, Weyl, and Majorana fermions [24]. In addition to these powerful experimental and theoretical developments, there have been more recently added to the list. One is the development of the methods for high precision measurements of the thermodynamic properties such as the compressibility, the chemical potential, the entropy, and the heat capacity for uniform $s$-wave Fermi superfluids [25, 26]. In recent developments, Ref. [26] has completely determined the universal thermodynamics of the interacting Fermi gas across the $s$ wave superfluid transition and has captured the onset of superlfuidity in the compressibility, chemical potential, entropy and the heat capacity. The question we address in this paper is what are the corresponding thermodynamic signatures for interacting ultra-cold fermions across the topological superfluid transitions to phases supporting Majorana fermions (2D) and Weyl fermions (3D). Our main conclusion is that among the various thermodynamic quantities, the isothermal compressibility and the chemical potential as a function of the artificial Zeeman field have the strongest signatures of the topological transitions. The signatures for the transition from the non-topological $s$-wave superfluid to the Majorana superfluid phase in $2 \mathrm{D}$ are stronger than the corresponding signatures in 3D where the transition is in the Weyl superfluid phase. The other thermodynamic quantities such as the pressure, entropy, and specific heat have only weak signatures of the topological transitions (that is, the transition between a non-topological superfluid and a topological superfluid as a function of the Zeeman field), even though they are useful to capture the topological superfluid to the normal phase (pair potential $\Delta=0$ ) transitions in both two and three dimensions.

In condensed matter systems, SOC, which results from the coupling between the spin degrees of freedom and the orbital motion of a quantum particle, plays an important role in understanding interesting physics associated with the topological quantum phenomena including the spin Hall effect [27, 28], topological insulators [29], and topological superconductors [30]. These phases generically arise as a result of topological quantum phase transitions (TQPT) in the appropriate systems. TQPTs separate distinct phases of matter which have exactly the same symmetries and thus are not associated with any spontaneous symmetry breaking in the underlying Hamiltonian. Yet, the phases separated by TQPTs have distinct topological properties which can often manifest themselves in the properties of the order parameter defects. For instance, it has been pointed out recently that spin-orbit 
coupled electron- or hole-doped semiconductor thin films (2D) or nanowires (1D) with proximity induced $s$-wave superconductivity and a suitably directed Zeeman field $(\Gamma)$ supports novel non-Abelian topological states with the order parameter defects supporting localized topological zero-energy excitations called Majorana fermions [31-36] above a critical Zeeman field $\left(\Gamma>\Gamma_{c}\right)$. These theoretical developments in the condensed matter system followed earlier similar proposals in the context of ultra-cold fermions in two dimensions in the presence of artificial SOC and a Zeeman field and with $s$-wave pairing interactions [22]. While the two dimensional cold fermion systems support the Majorana fermions in the order parameter defects (but are otherwise completely gapped in the bulk), the corresponding three dimensional systems have topologically protected gapless nodes in the bulk supporting the relativistic Weyl fermion excitations. With Rashba-type of SOC and an increasing strength of the Zeeman field, the three dimensional system supports a series of 3D topological quantum phase transitions [37] from a non-topological superfluid state with fully gapped fermionic excitations to a topological superfluid state with four protected Fermi points (i.e., gap nodes) and then to a second topological superfluid state with only two protected Fermi points [17]. Such 3D topological gapless superfluids with Weyl fermions, which result from quasiparticle excitations in a linearized Hamiltonian near the nodes, is different from the 2D fully gapped topological superfluids with Majorana fermions [18, 31]. Recently, similar Weyl fermion physics in an analogous 3D condensed matter system has also been discussed in the context of ferromagnetic superconductors [38]. The results on the thermodynamic signatures of the topological transitions to Majorana and Weyl superfluid phases discussed in this paper apply to both cold fermion and condensed matter systems although our focus will mostly be ultra-cold fermions where the methods for high precision measurements of the thermodynamic quantities have recently been successfully developed.

The paper is organized as follows. In Sec. II, we derive the mean-field order parameter and atom-number equations for ultra-cold fermions with Rashba SOC and a Zeeman field. We present the zero temperature phase diagram and the thermodynamic quantities as a function of interaction for both 2D and 3D Fermi superfluids from the self-consistent numerical calculations in Sec. III. Section IV provides the phase diagram and the corresponding thermodynamic properties at finite temperature. Section V consists of discussion and conclusions.

\section{MEAN-FIELD THEORY}

The system we consider is a uniform $s$-wave fermionic superfluid with the atom density $n=N / V$ in the presence of Rashba-type spin-orbit coupling (SOC) in the $x y$ plane and a Zeeman field along the $z$ direction. The dynamics of the Fermi gas can be described by the Hamiltonian $H=H_{0}+H_{\text {int }}$, where the single particle Hamiltonian

$$
H_{0}=\sum_{\gamma \gamma^{\prime}} \int d \mathbf{r} c_{\mathbf{r} \gamma}^{\dagger}\left[\xi I-i \hbar \alpha\left(\partial_{y} \sigma_{x}-\partial_{x} \sigma_{y}\right)+\Gamma \sigma_{z}\right]_{\gamma \gamma^{\prime}} c_{\mathbf{r} \gamma^{\prime}},
$$

where $\gamma=\uparrow, \downarrow$ are the pseudo-spin of the atoms, $\xi=$ $-\hbar^{2} \nabla^{2} / 2 m-\mu, \mu$ is the chemical potential, $\alpha$ is the Rashba SOC strength, $I$ is the $2 \times 2$ unit matrix, $\sigma_{i}$ is the Pauli matrix, $\Gamma$ is the strength of the Zeeman field, and $c_{\mathbf{r} \gamma}$ is the atom annihilation operator. $H_{\text {int }}=-g \int d \mathbf{r} c_{\mathbf{r} \uparrow}^{\dagger} c_{\mathbf{r} \downarrow}^{\dagger} c_{\mathbf{r} \downarrow} c_{\mathbf{r} \uparrow}$ is the $s$-wave scattering interaction with $g=4 \pi \hbar^{2} \bar{a}_{s} / m$, and the scattering length $\bar{a}_{s}$ can be tuned by the Feshbach resonance.

The grand partition function at temperature $T$ is $Z=$ $\int D[\psi, \bar{\psi}] \exp (-S[\psi, \bar{\psi}])$ with action

$$
S[\psi, \bar{\psi}]=\int d \tau d \mathbf{r}\left[\sum_{\gamma} \bar{\psi}_{\gamma}(\mathbf{r}, \tau) \frac{\partial}{\partial \tau} \psi_{\gamma}(\mathbf{r}, \tau)+\mathcal{H}(\mathbf{r}, \tau)\right] .
$$

Using the standard Hubbard-Stratanovich transformation that introduces the pairing potential $\Delta(\mathbf{r}, \tau)=g\left\langle\psi_{\downarrow}(\mathbf{r}, \tau) \psi_{\uparrow}(\mathbf{r}, \tau)\right\rangle$ and integrating over the fermion variables lead to the effective action

$$
S_{\mathrm{eff}}=\int d \tau d \mathbf{r}\left[\frac{|\Delta(\mathbf{r}, \tau)|^{2}}{g}-\frac{T}{2 V} \ln \operatorname{det} \frac{\mathbf{M}}{T}+\xi \delta\left(\mathbf{r}-\mathbf{r}^{\prime}\right)\right],
$$

where $\xi=-\hbar^{2} \nabla^{2} / 2 m-\mu$ and the matrix $\mathbf{M}$ is

$$
\mathbf{M}=\left(\begin{array}{cccc}
\partial_{\tau}+\tilde{\xi}_{\uparrow} & h_{\perp} & 0 & -\Delta \\
-h_{\perp}^{*} & \partial_{\tau}+\tilde{\xi}_{\downarrow} & \Delta & 0 \\
0 & \Delta^{*} & \partial_{\tau}-\tilde{\xi}_{\uparrow} & h_{\perp}^{*} \\
-\Delta^{*} & 0 & h_{\perp} & \partial_{\tau}-\tilde{\xi}_{\downarrow}
\end{array}\right),
$$

where $h_{\perp}=-i \hbar \alpha\left(\partial_{y}+i \partial_{x}\right)$ corresponds to the transverse component of the SOC field, $\Gamma$ to the parallel component with respect to the quantization axix $z$, and $\tilde{\xi}_{\uparrow, \downarrow}=\xi \pm \Gamma$.

To proceed, we use the saddle point approximation $\Delta(\mathbf{r}, \tau)=\Delta_{0}+\eta(\mathbf{r}, \tau)$, and write $\mathbf{M}=\mathbf{M}_{0}+\mathbf{M}_{F}$. The matrix $\mathbf{M}_{0}$ is obtained via the saddle point $\Delta(\mathbf{r}, \tau) \rightarrow \Delta$ which takes $\mathbf{M} \rightarrow \mathbf{M}_{0}$, and the functional matrix $\mathbf{M}_{F}=\mathbf{M}-\mathbf{M}_{0}$ depends only on $\eta(\mathbf{r}, \tau)$ and its Hermition conjugate. Thus, we write the effective action as $S_{\text {eff }}=S_{0}+S_{F}$. The first term is

$$
S_{0}=\frac{V}{T} \frac{|\Delta|^{2}}{g}-\frac{1}{2} \sum_{\mathbf{k}, i \omega_{n}, \eta, \lambda} \ln \left(\frac{i \omega_{n}-E_{\mathbf{k}, \eta}^{\lambda}}{T}\right)+\sum_{\mathbf{k}} \frac{\tilde{\xi}}{T},
$$

where $\omega_{n}=(2 n+1) \pi T$. Here $E_{\mathbf{k}, \eta}^{\lambda}$ are the eigenvalues of

$$
\mathbf{M}_{\mathbf{k}}=\left(\begin{array}{cccc}
\xi_{\mathbf{k}}+\Gamma & -h_{\perp}(\mathbf{k}) & 0 & -\Delta \\
-h_{\perp}^{*}(\mathbf{k}) & \xi_{\mathbf{k}}-\Gamma & \Delta & 0 \\
0 & \Delta^{*} & -\xi_{\mathbf{k}}-\Gamma & h_{\perp}(-\mathbf{k}) \\
-\Delta^{*} & 0 & h_{\perp}(-\mathbf{k}) & -\xi_{\mathbf{k}}+\Gamma
\end{array}\right),
$$

which describes the Hamiltonian of elementary excitations in the 4-component Nambu spinor $\Psi_{\mathbf{k}}=\left(c_{\mathbf{k} \uparrow}, c_{\mathbf{k} \downarrow}, c_{-\mathbf{k} \uparrow}^{\dagger}, c_{-\mathbf{k} \downarrow}^{\dagger}\right)^{\dagger}$. Here, $h_{\perp}(\mathbf{k})=\alpha\left(k_{y}+i k_{x}\right)$ and the quasiparticle excitation energy

$$
E_{\mathbf{k} \pm}^{\lambda}=\lambda \sqrt{\xi_{\mathbf{k}}^{2}+\alpha^{2} k_{\perp}^{2}+\Gamma^{2}+|\Delta|^{2} \pm 2 E_{0}}
$$


is the eigenvalue of $M_{\mathbf{k}}$, where $\lambda= \pm$ correspond to the particle and hole branches, $E_{0}=\sqrt{\Gamma^{2}\left(\xi_{\mathbf{k}}^{2}+|\Delta|^{2}\right)+\alpha^{2} k_{\perp}^{2} \xi_{\mathbf{k}}^{2}}$, and $k_{\perp}=\sqrt{k_{x}^{2}+k_{y}^{2}}$. For $\alpha=\Gamma=0$, Eq. (7) reduces to $E_{\mathbf{k}}^{\lambda}=\lambda \sqrt{\xi_{\mathbf{k}}^{2}+|\Delta|^{2}}$ in the standard BCS theory.

The saddle-point grand potential function $\Omega=-T \ln Z_{0}$ is

$$
\Omega=V \frac{|\Delta|^{2}}{g}-\frac{T}{2} \sum_{\mathbf{k}, \eta, \lambda} \ln \left(1+e^{-E_{\mathbf{k}, \eta}^{\lambda} / T}\right)+\sum_{\mathbf{k}} \xi_{\mathbf{k}}
$$

leading to the order parameter via minimization of $\Omega$ with respect to $\Delta$ :

$$
\frac{\Delta}{g}+\frac{1}{2 V} \sum_{\mathbf{k}, \eta, \lambda} f\left(E_{\mathbf{k}, \eta}^{\lambda}\right)\left(\frac{\partial E_{\mathbf{k}, \eta}^{\lambda}}{\partial \Delta^{*}}\right)=0
$$

where $f(x)=1 /(1+\exp (-x / T))$ is the Fermi-Dirac distribution function. For the case of $2 \mathrm{D}$ gas, Eq. (9) can be expressed as

$$
\sum_{\mathbf{k}, \eta}\left[\left(1-\eta \Gamma^{2} / E_{0}\right) \tanh \left(E_{\mathbf{k}, \eta}^{+} / 2 T\right) / 4 E_{\mathbf{k}, \eta}^{+}-\frac{1}{2 \epsilon_{\mathbf{k}}+E_{b}}\right]=0,
$$

where $E_{b}=\hbar^{2} / m a^{2}$ is the binding energy controlled by Feshbach resonances, and for $3 \mathrm{D}$ gas,

$$
\frac{V m}{4 \pi \hbar^{2} a_{s}}=-\sum_{\mathbf{k}, \eta}\left[\left(1-\eta \Gamma^{2} / E_{0}\right) \tanh \left(E_{\mathbf{k}, \eta}^{+} / 2 T\right) / 4 E_{\mathbf{k}, \eta}^{+}-\frac{1}{2 \epsilon_{\mathbf{k}}}\right] .
$$

The ultra-violet divergence at the large $\mathbf{k}$ in Eq. (9) has been regularized through subtracting the term $1 / 2 \epsilon_{\mathbf{k}}$ and $a_{s}$ is defined as the renormalized scattering length. The total number of atoms can be obtained from $N=-(\partial \Omega / \partial \mu)$ :

$$
N=\sum_{\mathbf{k}, \eta}\left[1+\left(\frac{\eta\left(\alpha^{2} k_{\perp}^{2}+\Gamma^{2}\right)}{E_{0}}-1\right) \frac{\xi_{\mathbf{k}}}{2 E_{\mathbf{k}, \eta}^{+}} \tanh \left(E_{\mathbf{k}, \eta}^{+} / 2 T\right)\right] .
$$

We self-consistently solve the order parameter equation (9) and the number equation (10) for different parameters $\left(\alpha K_{F}, \Gamma, v, T\right)$ for a fixed atom density $n$ to determine $\Delta$ and $\mu$. Here $v=1 / K_{F} a_{s}$ and $K_{F}=\left(3 \pi^{2} n\right)^{1 / 3}$ is the Fermi momentum for a non-interacting 3D Fermi gas with the same density at $\Gamma=\alpha=0$ and $v=E_{b}$ and $K_{F}=(2 \pi n)^{1 / 2}$ for a non-interacting 2D Fermi gas. The energy unit is chosen as the Fermi energy $E_{F}=\hbar^{2} K_{F}^{2} / 2 m$.

\section{TOPOLOGICAL PHASE TRANSITIONS DURING BCS-BEC CROSSOVER $(T=0)$}

\section{A. Phase diagram}

In Fig. 11 we show the mean-field zero temperature phase diagram as a function of the Zeeman field $\Gamma$ and the interaction parameter $v$ in the presence of Rashba SOC $\left(\alpha K_{F}=0.4\right)$.
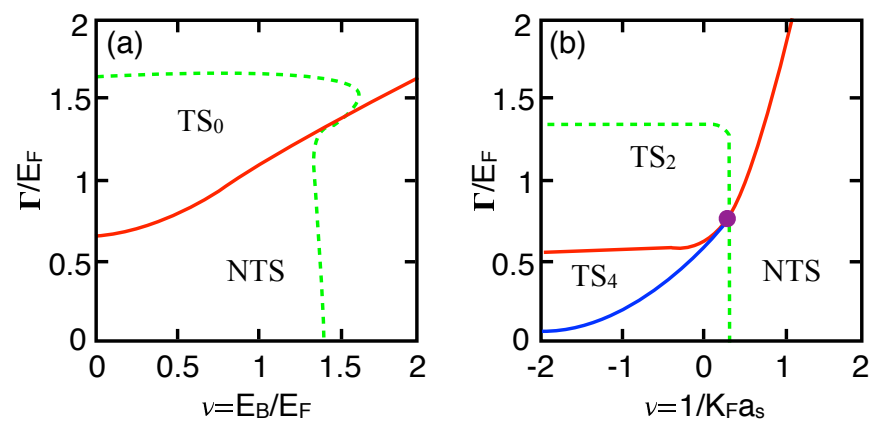

FIG. 1. (Color online) Plot of ( $\Gamma-v$ ) phase diagram for (a) 2D and (b) 3D Fermi gas at $T=0$ with $\alpha K_{F}=0.4$. Here, $v$ represents interaction parameters, $E_{B} / E_{F}$ for $2 \mathrm{D}$ and $1 / K_{F} a_{s}$ for $3 \mathrm{D}$ systems. We label fully gapped non-topological superfluid phase as NTS, fully gapped topological superfluid phase as $\mathrm{TS}_{0}$, and topological superfluids with 2 and 4 nodes as $\mathrm{TS}_{2}$ and $\mathrm{TS}_{4}$, respectively. Red and blue lines denote $\Gamma_{c}=\sqrt{\mu^{2}+\Delta^{2}}$ and $\Gamma_{\Delta}=\Delta$, respectively, where the topological phase transitions occur. Green dashed lines correspond to $\mu=0$, below which $\mu>0$ and above which $\mu<0$. Note that $\mathrm{TS}_{4}$ superfluid phase, which is characterized by 4 nodes along $z$-axis, arises when $\mu>0$ and merges at the tricritical point, which is indicated by purple circle in the phase diagram of 3D fermion superfluid.

We label the uniform topologically non-trivial $s$-wave superfluid phases with zero, one, two, and four nodal points as $\mathrm{TS}_{0}$, $\mathrm{TS}_{1}, \mathrm{TS}_{2}$, and $\mathrm{TS}_{4}$, respectively. For $2 \mathrm{D}$ Fermi gas as in Fig.1 (a), topologically non-trivial superfluid phase with one node, $\mathrm{TS}_{1}$, occurs only along $\Gamma=\Gamma_{c}=\sqrt{\mu^{2}+\Delta^{2}}$ line. Even though below and above $\Gamma=\Gamma_{c}$, the superfluid gap remains finite everywhere in the momentum space, thus the excitation spectrum is fully gapped, $\Gamma_{c}$ is a phase boundary between the nontopological superfluid (NTS) and non-trivial $\left(\mathrm{TS}_{0}\right)$ superfluid phases. Even though the $\mathrm{TS}_{0}$ phase is fully gapped, the phase is topologically non-trivial because in the presence of an order parameter defect such as a vortex, the vortex core supports a non-degenerate zero energy Majorana fermion excitation. The $\mathrm{TS}_{0}$ phase has recently received wide attention in condensed matter physics [31-36, 39-41] where 2D semiconducting thin films and 1D semiconducting nanowires with proximity induced $s$-wave superconductivity and a Zeeman field have been shown to support Majorana fermions in order parameter defects such as vortices and boundaries. Such localized Majorana fermion excitations are thought to support non-Abelian statistics which has been proposed as a key resource for faulttolerant topological quantum computation (TQC) [42]. 2D ultra cold fermionic gases with laser induced Rashba SOC and a Zeeman field in the $\mathrm{TS}_{0}$ phase can also support Majorana fermions in vortices and sample boundaries and thus can potentially act as a platform for fault tolerant TQC. In contrast, for 3D Fermi superfluid as in Fig. 1 (b), the blue line determined by the Chandrasekhar-Clogston like condition $\Gamma=\Delta$ is a topological phase boundary between fully gapped NTS $(\Gamma<\Delta)$ and gapless TS $(\Gamma>\Delta)$ phases. In addition, within the gapless TS phase, $\Gamma_{c}$ is a quantum critical point of the topological phase transition between the TS phases with two $\left(\mathrm{TS}_{2}\right)$ and four $\left(\mathrm{TS}_{4}\right)$ nodes. The fermions with linear dispersion relations near each node in the $\mathrm{TS}_{2}$ and $\mathrm{TS}_{4}$ phases are 
a manifestation of the relativistic Weyl fermions. The bulk nodal excitation spectra of these phases are also topologically protected and a topological invariant characterizing the nodal points (in the context of He3) was formulated by Volovik [37]. Recently, similar topologically protected Weyl fermion modes have been shown to exist also in condensed matter systems such as ferromagnetic superconductors [38].

\section{B. Chemical potential}

Chemical potential can be calculated by solving the order parameter equation with fixing the total number of the system self-consistently. With Zeeman field $\Gamma$ and interaction $v$ as tuning parameters, one can have chemical potential as a function of $\Gamma$ and $v$, that is, $\mu=\mu(\Gamma, v)$.

In Fig. 2, we show the chemical potential as a function of $\Gamma$ for a given interaction $v$ and SOC $\alpha K_{F}$. Fig. 2 (a) and (b) are plots of the chemical potential $\mu$ and its derivative with respect to $\Gamma, \frac{d \mu}{d \Gamma}$, for two dimensional Fermi gas at $E_{b}=0.5 E_{F}$ and $\alpha K_{F}=0.4$. In the side of low Zeeman field $\Gamma$, where $\Gamma<\Gamma_{c}=\sqrt{\mu^{2}+\Delta^{2}}$ and the system is in the non-topological superfluid phase (NTS), $\mu$ is slowly varying as $\Gamma$ increases. In the region of strong Zeeman field, where $\Gamma>\Gamma_{c}$ and the system is in a gapped $\mathrm{TS}_{0}$ phase, $\mu$ decreases fast with increasing $\Gamma$. Near the quantum critical point $\Gamma_{c}$, the slope changes discontinuously as can be seen clearly in the plot of $\frac{d \mu}{d \Gamma}$ in Fig. 2 (b), indicating the change in the curvature.

As for the 3D Fermi superfluids, topological phase transition from a fully gapped NTS to a gapless $\mathrm{TS}_{4}$ state occurs at Zeeman field $\Gamma=\Gamma_{\Delta}=\Delta$, and $\mathrm{TS}_{4}$ to $\mathrm{TS}_{2}$ phase transition occurs at $\Gamma=\Gamma_{c}=\sqrt{\mu^{2}+\Delta^{2}}$. Fig. 2 (c) and (d) illustrate $\mu$ as a function of $\Gamma$ at unitarity $\left(1 / K_{F} a_{s}=0\right)$ with the same SOC strength as that in $2 \mathrm{D}$ fermion gas. Even though the signatures of topological phase transitions across the phase boundaries are weaker than the one for the 2D Fermi system, $\mu$ in the fully gapped NTS phase $\left(\Gamma<\Gamma_{\Delta}\right)$ increases with increasing $\Gamma$, while $\mu$ decreases with increasing $\Gamma$ when it is in the $\mathrm{TS}_{2}$ phase $\left(\Gamma>\Gamma_{c}\right)$. The curvature of the chemical potential versus the Zeeman field changes as the system moves from the gapped to the gapless phases and vice versa. In contrast to the 2D Fermi superfluid, however, $\frac{d \mu}{d \Gamma}$ plot doesn't exhibit a cusp at the phase boundary because of the existence of the $\mathrm{TS}_{4}$ phase surrounded by the gapped NTS and gapless $\mathrm{TS}_{2}$ phases. It is when $\mu=0$ that the two distinct critical points $\Gamma_{\Delta}$ and $\Gamma_{c}$ merge $\left(\Gamma_{\Delta}=\Gamma_{c}\right)$ and a tricritical point occurs, leading to the vanishing of the $\mathrm{TS}_{4}$ phase and the emergence of the cusp in $\frac{d \mu}{d \Gamma}$ even in a 3D Fermi superfluid system.

\section{Compressibility}

The compressibility can be obtained from the knowledge of the pressure, as defined by, $\kappa_{T}=-(1 / V)(\partial p / \partial V)$. But in the grand-canonical ensemble, where we need to fix the average number of particles, the isothermal compressibility can be
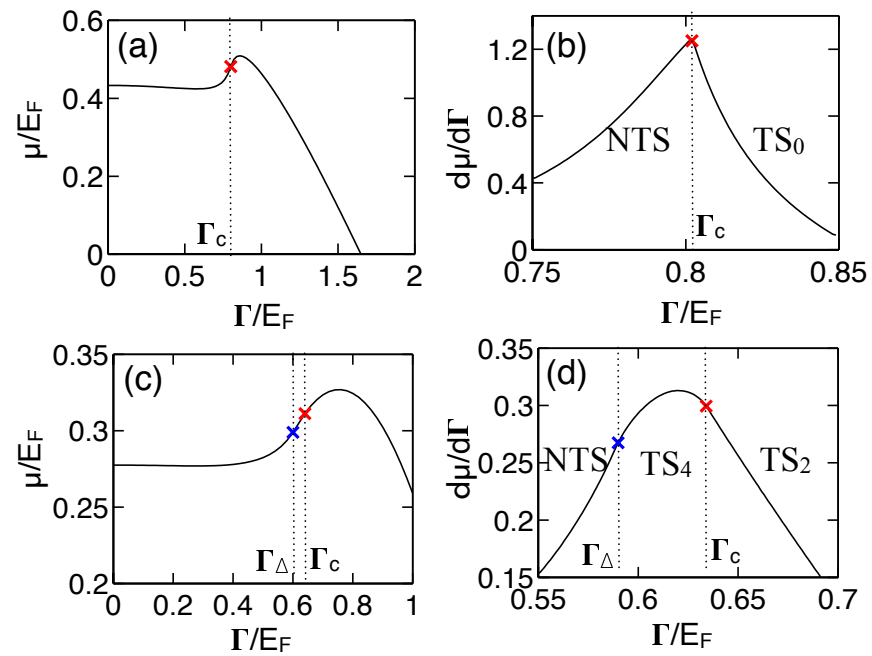

FIG. 2. (Color online) Plot of chemical potential $\mu$ and its derivative with respect to $\Gamma, \frac{d \mu}{d \Gamma}$ for (a)-(b) $2 \mathrm{D}$ Fermi gas at $E_{b}=0.5 E_{F}$ and (c)-(d) 3D Fermi gas at $1 / K_{F} a_{s}=0$ with $\alpha K_{F}=0.4$. Blue and red crosses indicate the critical points $\Gamma_{\Delta}=\Delta$ and $\Gamma_{c}=\sqrt{\mu^{2}+\Delta^{2}}$, respectively. One finds that fully gapped NTS phase occupies at $\Gamma<$ $\Gamma_{c}$ for $2 \mathrm{D}$ and $\Gamma<\Gamma_{\Delta}$ for $3 \mathrm{D}$ gases, respectively.

directly written as,

$$
\kappa_{T}=\frac{1}{N^{2}}\left(\frac{\partial N}{\partial \mu}\right)_{T, V}
$$

In the superfluid state, we need to consider $\mu$ dependence of $\Delta$. From the order parameter equation $(\partial \Omega / \partial \Delta)=0$, we have $(\partial / \partial \mu)(\partial \Omega / \partial \Delta)=0$, leading to

$$
\left(\frac{\partial \Delta}{\partial \mu}\right)_{T, V}=-\left(\frac{\partial N}{\partial \Delta}\right)_{T, V, \mu}\left(\frac{\partial^{2} \Omega}{\partial \Delta^{2}}\right)_{T, V, \mu}^{-1}
$$

Thus the isothermal compressibility in the superfluid phase can be written as

$$
\kappa_{T}=\frac{1}{N^{2}}\left[\left(\frac{\partial N}{\partial \mu}\right)_{T, V, \Delta}-\left(\frac{\partial N}{\partial \Delta}\right)_{T, V, \mu}^{2}\left(\frac{\partial^{2} \Omega}{\partial \Delta^{2}}\right)_{T, V, \mu}^{-1}\right]
$$

In Fig. 3, we present the isothermal compressibility $\kappa_{T}$ as a function of $\Gamma$ for a given interaction $v$ at $T=0$ with $\alpha K_{F}=0.4$ to investigate the signature of the topological phase transitions. Since $\kappa_{T}$ is related to the first derivative of the total number $N$ with respect to $\mu$, that is, $\left.\kappa_{T} \sim \frac{\partial N}{\partial \mu}\right|_{T, V}$, it is expected to show the signatures of the topological phase transitions as seen in the plots of $\mu$ in Fig. 2. In Fig. 3, $\kappa_{T}$ shows a decreasing trend in the superfluid phase with increasing $\Gamma$, while it is an increasing function of $\Gamma$ in normal phase as will be shown in Fig. 5] For 2D uniform Fermi superfluids, at the critical Zeeman field $\Gamma_{c}$, a sharp peak emerges as shown in Fig.3(a). This peak in the compressibility corresponds to the phase boundary between the $2 \mathrm{D}$ gapped NTS and the $2 \mathrm{D}$ gapped $\mathrm{TS}_{0}$ phases. In our calculations we find that the compressibility provides the strongest signature of the phase transition to the 2D topological phase with the Majorana fermion excitations. In 2D at 

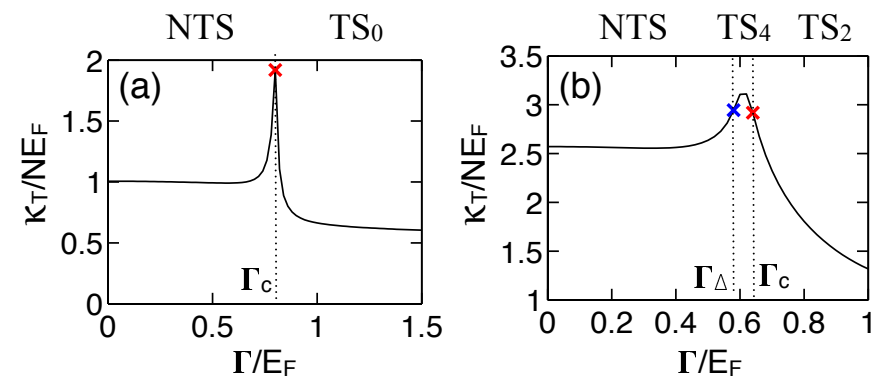

FIG. 3. (Color online) Plot of isothermal compressibility $\kappa_{T}$ as a function of $\Gamma$ for (a) 2D Fermi gas at $E_{b}=0.5 E_{F}$ and (b) 3D Fermi gas at $1 / K_{F} a_{s}=0$ with $\alpha K_{F}=0.4$. Blue and red crosses indicate the critical points $\Gamma_{\Delta}=\Delta$ and $\Gamma_{c}=\sqrt{\mu^{2}+\Delta^{2}}$, respectively, same as in Fig.11and Fig. 2

zero temperature there is no additional phase transition from the $\mathrm{TS}_{0}$ phase to the normal phase at higher values of $\Gamma$. This is because, with a finite Rashba SOC, the superfluid pair potential always remains non-zero even at large values of the Zeeman field. When the temperature is included as another parameter on the phase diagram, a normal phase with $\Delta=0$ emerges even in $2 \mathrm{D}$ (see below).

As for the 3D case, Fig. 3 (b) shows that $\kappa_{T}(\Gamma)$ again decreases with increasing $\Gamma$ in the superfluid phase, and shows the signature of the topological phase transitions by a smoothly peaked curve near $\Gamma=\Gamma_{c}$. As already seen in the plot of $\mu$ vs. $\Gamma, \kappa_{T}$ of 3D fermion system in the $\mathrm{TS}_{4}$ phase, which is sandwiched between the gapped NTS and gapless $\mathrm{TS}_{2}$ phases, is a fast varying function of $\Gamma$, while the compressibility in the gapped NTS phase is slowly varying.

\section{THERMODYNAMIC SIGANTURES $(T \neq 0)$}

Experimentally, the thermodynamic properties of a given substance is determined by measuring an equation of state (EoS), such as the pressure $p(\mu, T)$ as a function of the chemical potential $\mu$ and the temperature $T$. Equivalently, replacing the pressure by the density $n=\left(\frac{\partial p}{\partial \mu}\right)_{T}$, one can determine the density EoS, $n(\mu, T)$. The local gas density $n(V)$ can be measured [26] as a function of the local trapping potential $V$ from in situ absorption images of a trapped strongly interacting Fermi gas at a Feshbach resonance. From the definition of the compressibility $\kappa_{T}=\frac{1}{N^{2}}\left(\frac{\partial N}{\partial \mu}\right)_{T}$, the chemical potential as a function of temperature $T$ is attainable experimentally without numerical derivatives of the data [26]. Then, from the energy, pressure, and the chemical potential, the entropy $S=\frac{1}{T}(E+p V-\mu N)$ and the specific heat $c_{V}$ can be obtained as a function of temperature $T$.

\section{A. Thermodynamic Properties}

From the mean-field grand partition function

$$
Z=\operatorname{Tr}\left(e^{-(H-\mu N) / T}\right)
$$

we can calculate the corresponding grand potential function $\Omega=-T \ln Z=\langle H\rangle-T S-\mu\langle N\rangle=-p V$, where $S$ is the entropy and $p$ is the pressure of the system. The differential of the grand potential function is

$$
d \Omega=-S d T-p d V-\langle N\rangle d \mu,
$$

allowing us to obtain interesting thermodynamic properties. The explicit expression of $\Omega$ in terms of the Hamiltonian under consideration is

$$
\Omega=-\frac{T}{2} \sum_{\mathbf{k}, \eta, \lambda} \ln \left(1+e^{-E_{\mathbf{k}, \eta}^{\lambda} / T}\right)+\sum_{\mathbf{k}} \xi_{\mathbf{k}}+V \frac{|\Delta|^{2}}{g}
$$

Then, the pressure of the ultracold Fermi gas can be obtained through the grand potential function via $p=-\Omega / V$. In the thermodynamic limit, it reads

$$
p=-\frac{T}{2} \sum_{\eta, \lambda} \int \frac{d^{D} k}{(2 \pi)^{D}} \ln \left(1+e^{-E_{\mathbf{k}, \eta}^{\lambda} / T}\right)+\int \frac{d^{D} k}{(2 \pi)^{D}} \xi_{\mathbf{k}}+\frac{|\Delta|^{2}}{g}
$$

The entropy can be obtained by

$$
S=-\left(\frac{\partial \Omega}{\partial T}\right)_{\mu, V} .
$$

Then, the heat capacity can be obtained from the knowledge of the entropy.

$$
c_{V}=\left(\frac{\partial S}{\partial T}\right)_{\mu, V} .
$$

In the superfluid phase, $\Delta$ depends on temperature $T$ as

$$
\left(\frac{\partial \Delta}{\partial T}\right)_{\mu, V}=-\left(\frac{\partial S}{\partial \Delta}\right)_{T, V, \mu}\left(\frac{\partial^{2} \Omega}{\partial \Delta^{2}}\right)_{T, V, \mu}^{-1},
$$

leading to the specific heat in the superfluid phase

$$
c_{V}=\left(\frac{\partial S}{\partial T}\right)_{\mu, V, \Delta}-\left(\frac{\partial S}{\partial \Delta}\right)_{T, V, \mu}^{2}\left(\frac{\partial^{2} \Omega}{\partial \Delta^{2}}\right)_{T, V, \mu}^{-1} .
$$

\section{B. Finite Temperature Phase Diagram}

Fig. 4 presents mean-field $(T-\Gamma)$ phase diagram for $2 \mathrm{D}$ and 3D homogeneous Fermi gas in the presence of Rashba-type of spin-orbit coupling. We choose the interaction parameter $v$ as a two-body binding energy $E_{b}=0.5 E_{F}$ for 2D Fermi gas (a), while $1 / K_{F} a_{s}=0$ for 3D Fermi gas (b) with Rashba SOC $\alpha K_{F}=0.4$. With increasing temperature $T$, the superfluid order parameter $\Delta$ decreases and undergoes the second order phase transition to normal phase $(\Delta=0)$ denoted as $\mathrm{N}$ at $T=$ $T_{c}$.

In Fig. 4 (a), within the superfluid phase of 2D fermion gas, topological phase transition between the gapped NTS phase $\left(\Gamma<\Gamma_{c}\right)$ and the gapped $\mathrm{TS}_{0}$ phase $\left(\Gamma>\Gamma_{c}\right)$ occurs at $T=T_{1}<T_{c}$ indicated with a red line. In addition, the $\mathrm{TS}_{0}$ 
(a)

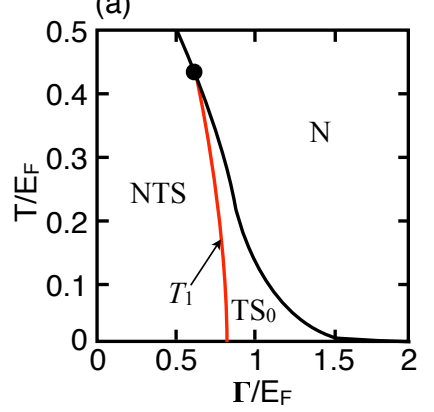

(b)

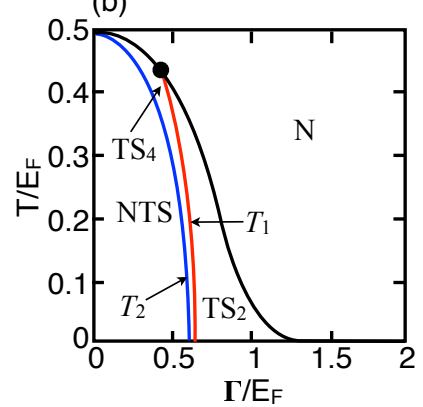

FIG. 4. (Color online) Plot of $(\Gamma-T)$ phase diagrams for (a) 2D Fermi gas at $E_{b}=0.5 E_{F}$ and (b) $3 \mathrm{D}$ Fermi gas at $1 / K_{F} a_{s}=0$ with $\alpha K_{F}=0.4$. Red and blue lines denote $\Gamma_{c}=\sqrt{\mu^{2}+\Delta^{2}}$ and $\Gamma_{\Delta}=\Delta$, respectively. Black lines represent $\Gamma_{0}$ above which the order parameter vanishes, or $\Delta=0$. The tricritical temperatures, at which $\Gamma_{c}$ meets with $\Gamma_{0}$ (black circles), for 2D and 3D gases are $T=0.43 E_{F}$ at $\Gamma_{c}=\Gamma_{0}=0.62 E_{F}$ and $T=0.44 E_{F}$ at $\Gamma_{c}=\Gamma_{0}=0.42 E_{F}$, respectively.

phase vanishes at the tricritical temperature $T_{1}=T_{c}=0.43 E_{F}$ with our choice of parameters $E_{b}$ and $\alpha$. In Fig. 4( 4 (b), $T_{2}$ (blue line) determined by the Chandrasekhar-Clogston-like condition, $\Gamma=\Delta$, is a phase boundary between the NTS phase $\left(T<T_{2}\right)$ and the $\mathrm{TS}_{4}$ phase $\left(T_{2}<T<\min \left[T_{c}, T_{1}\right]\right)$. It is when $\Gamma=0$ that $T_{2}=T_{c}$ occurs and the system above $T_{c}$ becomes the normal state $(\Delta=0)$. Thus, for $\Gamma=0$, there is only one finite temperature phase transition at $T_{c}\left(=T_{2}\right)$ which is a transition between the non-topological superfluid (NTS) $\left(T<T_{c}\right)$ and the normal state $\left(T>T_{c}\right)$. In a similar manner as in the 2D system, $\mathrm{TS}_{4}$ phase vanishes at finite $\Gamma$, where $T_{c}=T_{1}=0.43 E_{F}$ at unitarity $\left(1 / K_{F} a_{s}=0\right)$.

\section{Thermodynamic Quantities as a function of temperature}

Since we learned that the chemical potential and the isothermal compressibility at zero temperature can show the evidence of the topological phase transitions as a function of the tuning parameters such as the Zeeman field $\Gamma$ and the interaction parameter $v$, we first consider below the chemical potential and the compressibility at finite temperatures.

In Fig. 5, we show the chemical potential and isothermal compressibility as a function of the reduced Zeeman field $\Gamma / \Gamma_{0}$ for $2 \mathrm{D}$ on the left column and $3 \mathrm{D}$ on the right column at both $T>T_{1}$ (dashed) and $T<T_{1}$ (solid). We choose the Rashba SOC $\alpha K_{F}=0.4$ and the interaction parameters $E_{b}=0.5 E_{F}$ for $2 \mathrm{D}$ and $1 / K_{F} a_{s}=0$ for 3D Fermi gases, respectively. Fig. 5 (a) and (b) show that, at low temperatures, $T<T_{1}, \kappa_{T}$ for both 2D and 3D fermions exhibit peak at or near the topological critical point, and a discontinuity at the phase transition to the normal state at $\Gamma=\Gamma_{0}$. Meanwhile, at high temperatures (dashed), $T>T_{1}$, it is hard to see the signature of the topological phase transition between the gapped NTS and gapless $\mathrm{TS}_{4}$ phases. We notice that as temperature increases $\kappa_{T}$ increases in the superfluid phase, while it decreases in the normal phase. Fig. 51(c) and (d) are plots of both $2 \mathrm{D}$ and $3 \mathrm{D}$ chemical potentials as a function of $\Gamma / \Gamma_{0}$
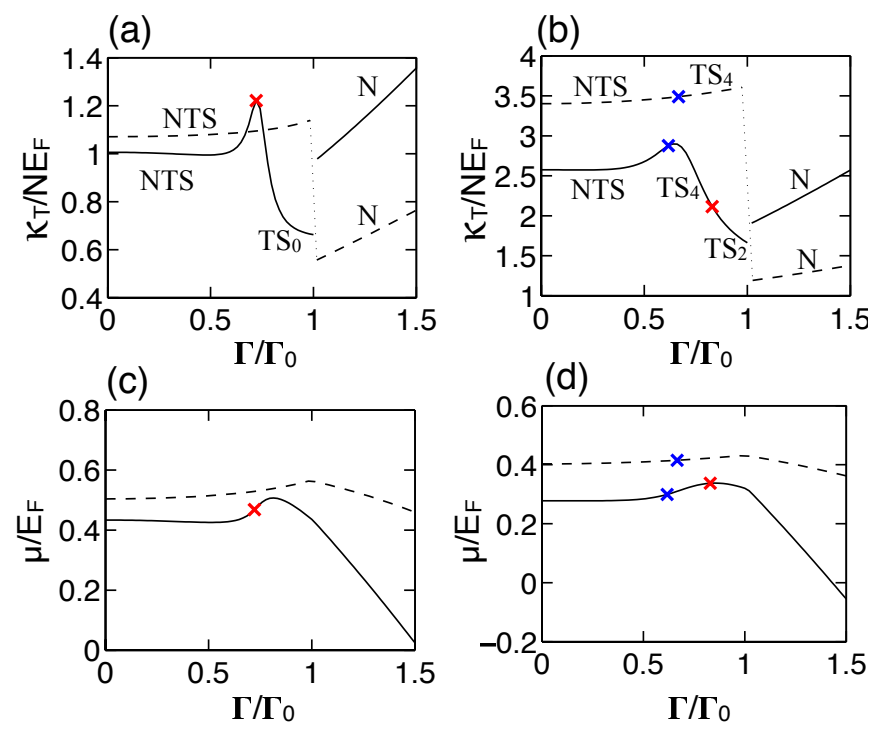

FIG. 5. (Color online) Plot of isothermal compressibility $\kappa_{T}$ and chemical potential $\mu$ as a function of $\Gamma / \Gamma_{c}$ for (a),(c) 2D and (b),(d) 3D Fermi gas at temperature $T=0.1 E_{F}$ (solid) and $T=0.45 E_{F}$ (dashed). We indicates topological phase transitions with red $\left(\mathrm{TS}_{4}\right.$ to $\mathrm{TS}_{2}$ ) and blue (NTS to $\mathrm{TS}_{4}$ ) crosses within the uniform superfluid phases. One finds that normal $(\mathrm{N})$ to superfluid phase transitions occur at $\Gamma_{0}$ and are characterized by the discontinuity of the isothermal compressibility $\kappa_{T}$, while the chemical potential $\mu$ remains a continuous function of $\Gamma$ as well as a function of $T$.

for $T=0.1 E_{F}$ (solid) and $T=0.45 E_{F}$ (dashed), respectively. It shows that $\mu$ is an increasing function of $T$ and $\mu$ in the superfluid phase is varying slowly compared with that in normal phase. Even though the signatures of the topological transition, which are strong at zero temperature, are weakened by the thermal fluctuations, the characteristic features for the variations of the thermodynamic quantities near the phase transitions remain the same.

In Fig. 6, we present the other relevant thermodynamic quantities in 2D and 3D Fermi gas, such as pressure $p$, entropy $S$, and specific heat $c_{V}$, which can be observed experimentally, as a function of $\Gamma / \Gamma_{0}$ at $T=0.1 E_{F}$ (solid) and $T=0.45 E_{F}$ (dashed). We choose $E_{b}=0.5 E_{F}$ for $2 \mathrm{D}$ and $1 / K_{F} a_{s}=0$ for 3D Fermi system with Rashba SOC $\alpha K_{F}=0.4$. The main conclusion from these calculations is that the signatures of the topological phase transitions are much weaker in these quantities than those in the case of ordinary phase transitions from the ordinary $s$-wave superfluid to normal phases.

\section{v. CONCLUSIONS}

In summary, we have investigated the two- and threedimensional uniform fermion $s$-wave superfluids in the presence of Rashba spin-orbit coupling together with Zeeman field as they undergo topological phase transitions as a function of the interaction parameter $v$ and Zeeman field $\Gamma$ at zero temperature and as a function of Zeeman field $\Gamma$ at finite temperatures. In 2D $s$-wave Fermi gas, for $\Gamma=\Gamma_{c}=\sqrt{\mu^{2}+\Delta^{2}}$ the 

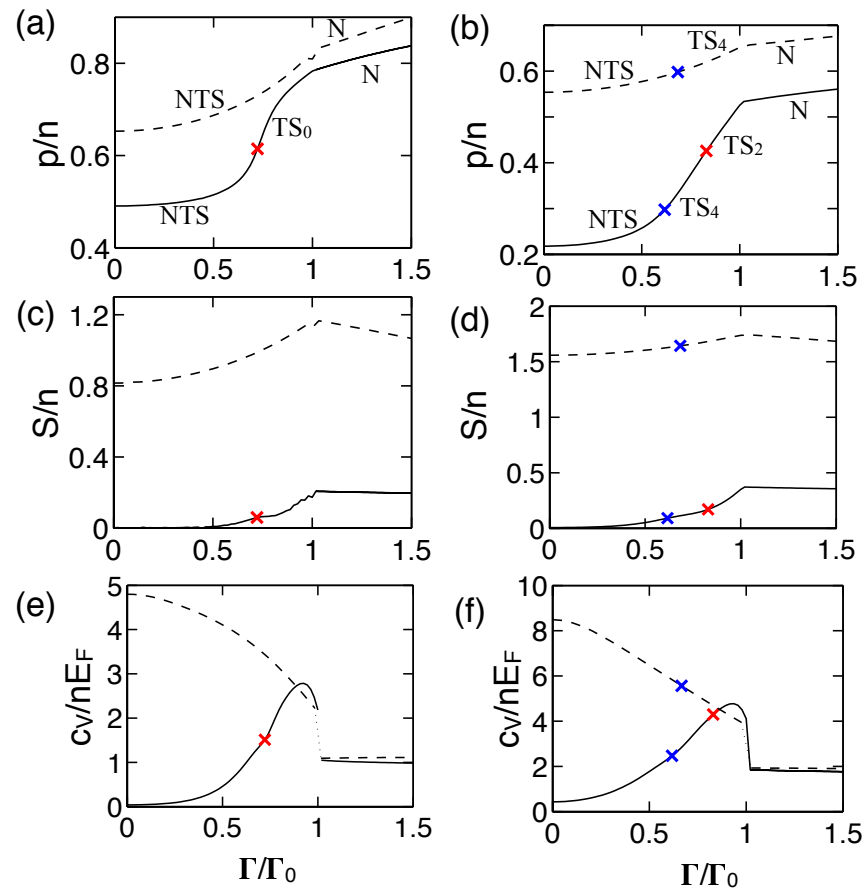

FIG. 6. (Color online) Plot of pressure $p$, entropy $S$, and specific heat $c_{V}$ as a function of $\Gamma / \Gamma_{0}$, where $\Gamma_{0}$ corresponds to the Zeeman field at $\Delta=0$, for (a),(c),(d) 2D Fermi gas at $E_{b}=0.5 E_{F}$ and (b),(d),(f) 3D Fermi gas at $1 / K_{F} a_{s}=0$ at $T=0.1 E_{F}$ (solid) and $T=0.45 E_{F}$ (dashed). We used the red and blue crosses for $\Gamma_{c}$ and $\Gamma_{\Delta}$, respectively. One notices that the signatures of the normal $(\mathrm{N})$ to superfluid phase transitions are visible in both 2D and 3D Fermi gases, characterized by the suppression of $p$ and $S$ in the superfluid state and the discontinuity of $c_{V}$. On the other hand, it is difficult to observe the topological phase transitions even with some weak signatures.

superfluid phase is identified with a gapless topological superfluid (with one node at $k=0$ ), while it becomes a fully gapped non-topological superfluid phase at $\Gamma<\Gamma_{c}$ and a fully gapped topological superfluid phase $\mathrm{TS}_{0}$, capable of supporting Majorana fermion excitations at order parameter defects such as vortices, at $\Gamma>\Gamma_{c}$. The $\mathrm{TS}_{0}$ phase has recently received wide attention in condensed matter physics [31-36, 39-41] where 2D semiconducting thin films and 1D semiconducting nanowires with proximity induced $s$-wave superconductivity and a Zeeman field have been shown to support Ma- jorana fermions in order parameter defects such as vortices and boundaries. Such localized Majorana fermion excitations are thought to support non-Abelian statistics which has been proposed as a key resource for fault-tolerant topological quantum computation (TQC) [42]. In contrast, 3D $s$-wave Fermi gas is a fully gapped non-topological superfluid at $\Gamma<\Delta$, becomes a gapless topological superfluid with four topologically protected Weyl fermion points [37] at $\Delta<\Gamma<\Gamma_{c}$ and another topological superfluid with two topologically protected Weyl fermion points at $\Gamma>\Gamma_{c}$. With our calculated mean-field phase diagrams in both $2 \mathrm{D}$ and $3 \mathrm{D}$ we investigated the thermodynamic properties across the relevant topological phase transitions, including the isothermal compressibility, the chemical potential, the pressure, the entropy, and the specific heat as a function of the Zeeman field $\Gamma$ and the interaction parameter $v$. In particular, the isothermal compressibility of the system supporting the Majorana fermions, which is the 2D fermion gas with Rashba SOC together and a perpendicular Zeeman field, reveals the topological phase transition via a strong peak at the critical point $\Gamma=\Gamma_{c}$ (Fig. 3). Furthermore, the phase boundary can also be identified by the chemical potential through the change of the curvature as a function of $\Gamma$ (Fig. 2). For the 3D Fermi gas, the topological phase transition from the fully gapped non-topological phase to the gapless topological phases can be probed by the position of the peak in the isothermal compressibility as a function of $\Gamma$, even if the signatures of the topological transitions are weaker than those in the 2D system. The signatures of the topological phase transitions at low temperature disappear as the temperature goes above the critical temperature $T_{1}$. The other thermodynamic quantities, such as the pressure, the entropy, and the specific heat, have only weak signatures of the topological phase transitions (that is, the transition between a conventional (non-topological) superfluid and a topological superfluid such as the Majorana and the Weyl phases), while these are useful quantities to identify the phase transitions from the normal phase to the topological superfluid phases with decreasing values of the Zeeman field.

\section{ACKNOWLEDGMENTS}

This work is supported by AFOSR (FA9550-13-1-0045), DARPA MTO (FA9550-10-1-0497), NSF-PHY (1104546), and ARO (W911NF-12-1-0334). and
[1] C. Chin et al., Rev. Mod. Phys. 82, 1225 (2010).

[2] S. Giorgini et al., Rev. Mod. Phys. 80, 1215 (2008).

[3] C. A. Regal, M. Greiner, and D. S. Jin, Phys. Rev. Lett. 92, 040403 (2004); M. W. Zwierlein et al., ibid. 92, 120403 (2004); M. Bartenstein et al., ibid. 92, 120401 (2004).

[4] J. T. Stewart et al., Phys. Rev. Lett. 104, 235301 (2010).

[5] M.W. Zwierlein et al., Nature (London) 435, 1047 (2005).

[6] M. W. Zwierlein et al., Science 311, 492 (2006).

[7] G. B. Partridge et al., Science 311, 503 (2006).

[8] Y. Liao et al., Nature (London) 467, 567 (2010).
[9] K. Martiyanov, V. Makhalov, and A. Turlapov, Phys. Rev. Lett. 105, 030404 (2010).

[10] M. Feld et al., Nature (London) 480, 75 (2011).

[11] Y.-J. Lin, K. Jimenez-Garcia, and I. B. Spielman, Nature (London) 471, 83 (2011).

[12] J.-Y. Zhang, S.-C. Ji, Z. Chen, L. Zhang, Z.-D. Du, B. Yan, G.S. Pan, B. Zhao, Y.-J. Deng, H. Zhai, S. Chen, and J.-W. Pan, Phys. Rev. Lett. 109, 115301 (2012).

[13] C. Qu, C. Hamner, M. Gong, C. Zhang, P. Engels, arXiv:1301.0658 
[14] P. Wang, Z.-Q. Yu, Z. Fu, J. Miao, L. Huang, S. Chai, H. Zhai, J. Zhang, Phys. Rev. Lett. 109, 095301 (2012).

[15] L.W. Cheuk, et al., Phys. Rev. Lett. 109, 095302 (2012).

[16] M. Chapman and C. Sá de Melo, Nature (London) 471, 41 (2011); J. P. Vyasanakere, S. Zhang, and V. B. Shenoy, Phys. Rev. B. 84, 014512 (2011); H. Hu, L. Jiang, X. J. Jiu, and H. Pu, Phys. Rev. Lett. 107, 195304 (2011); Z.-Q. Yu and H. Zhai, Phys. Rev. Lett. 107, 195305 (2011); M. Iskin and A. L. Subasi, Phys. Rev. Lett. 107, 050402 (2011); G. Chen, M. Gong, and C. Zhang, Phys. Rev. A 85, 013601 (2011); L. Han, C. A. R. Sá de Melo, Phys. Rev. A 85, 011606(R) (2012); K. Seo, L. Han, and C. A. R. Sá de Melo, Phys. Rev. A 85, 033601 (2012); L. Han, C. A. R. Sá de Melo, arXiv:1206.4984v1; Z. Zheng, M. Gong, X. Zhou, C. Zhang, and G.-C. Guo, arXiv:1208.2029.

[17] M. Gong, S. Tewari, and C. Zhang, Phys. Rev. Lett. 107, 195303 (2011).

[18] M. Gong, G. Chen, S. Jia, and C. Zhang, Phys. Rev. Lett. 109, 105302 (2012).

[19] K. Seo, L. Han, and C. A. R. Sá de Melo, Phys. Rev. Lett. 109, 105303 (2012)

[20] Y. A. Bychkov and E. I. Rashba, J. Phys. C 17, 6029 (1984).

[21] G. Dresselhaus, Phys. Rev. 100, 580 (1955).

[22] C. Zhang, S. Tewari, R. M. Lutchyn, S. Das Sarma., Phys. Rev. Lett. 101, 160401 (2008).

[23] M. Sato, Y. Takahashi, S. Fujimoto, Phys. Rev. Lett. 103, 020401 (2009)

[24] P. B. Pal, arXiv: 1006.1718

[25] N. Navon et al., Science 328, 729 (2010).
[26] M. J. H. Ku, A. T. Sommer, L. W. Cheuk, and M. W. Zwierlein, Science 335, 563 (2012).

[27] N. Nagaosa, J. Sinova, S. Onoda, A. H. MacDonald, and N. P. Ong, Rev. Mod. Phys. 82, 1539 (2010).

[28] D. Xiao, M.-C. Chang, and Q. Niu, Rev. Mod. Phys. 82, 1959 (2010).

[29] M. Z. Hasan and C. L. Kane, Rev. Mod. Phys. 823045 (2010).

[30] X.-L. Qi and S.-C. Zhang, Rev. Mod. Phys. 83, 1057 (2011).

[31] J. D. Sau, R. M. Lutchyn, S. Tewari, and S. Das Sarma, Phys. Rev. Lett. 104, 040502 (2010).

[32] J. D. Sau, S. Tewari, R. M. Lutchyn, T. Stanescu, S. Das Sarma, Phys.Rev.B 82, 214509 (2010)

[33] R. M. Lutchyn, J. D. Sau, and S. Das Sarma, Phys. Rev. Lett. 105, 077001 (2010).

[34] Y. Oreg, G. Refael, and F. von Oppen, Phys. Rev. Lett. 105, 177002 (2010).

[35] L. Mao, J. Shi, Q. Niu, C. Zhang, Phys. Rev. Lett. 106, 157003 (2011).

[36] L. Mao, M. Gong, E. Dumitrescu, S. Tewari, C. Zhang, Phys. Rev. Lett. 108, 177001 (2012)

[37] G. E. Volovik, The Universe in Helium Droplet (Clarendon Press, Oxford, 2003).

[38] J. D. Sau and S. Tewari, Phys. Rev. B 86, 104509 (2012).

[39] V. Mourik, K. Zuo, S. M. Frolov, S. R. Plissard, E. P. A. M. Bakkers and L. P. Kouwenhoven, Science 336, 1003 (2012).

[40] M. T. Deng, C. L. Yu, G. Y. Huang, M. Larsson, P. Caroff, H. Q. Xu, arXiv:1204.4130

[41] A. Das, Y. Ronen, Y. Most, Y. Oreg, M. Heiblum, H. Shtrikman, arXiv:1205.7073

[42] C. Nayak et al., Rev. Mod. Phys. 80, 1083 (2008). 